Mark Hieke'
Johannes Ruland'
Harald Anlauf'
Hermann Nirschl'
'Universität Karlsruhe, Institut
für Mechanische
Verfahrenstechnik und
Mechanik, Karlsruhe,
Germany.

Mark Hieke

Johannes Ruland'

Harald Anlauf'

'Universität Karlsruhe, Institut

für Mechanische

Mechanik, Karlsruhe,

Cermany.

\section{Research Article \\ Analysis of the Porosity of Filter Cakes Obtained by Filtration of Colloidal Suspensions}

\begin{abstract}
The filtration of suspensions in the colloidal regime is part of many processes. It is necessary due to the widespread use of nano-size particles in many applications. In nano-scale particle systems, the huge specific surface of the particles is dominating the processes. The question is whether the well-known filter theory is applicable for such type of products. The experiments in this study were conducted by gas-driven filtration. The analysis of the filter cake was done globally according to the Darcy equation and locally by the use of magnetic resonance imaging (MRI) techniques. Due to the very high resistances during filtration for colloidal suspensions, comparatively thin filter cakes with an approximate height of $0.5-3 \mathrm{~mm}$ were investigated. The overall porosities determined by the different techniques are in good agreement. The MRI technique allows an in situ measurement of the porosity gradient within a filter cake formed by compressible material.
\end{abstract}

Keywords: Filter cake structure, Filtration, Magnetic resonance imaging (MRI), Porosity

Received: November 19, 2008; accepted: January 16, 2009

DOI: $10.1002 /$ ceat.200800609

\section{Introduction}

The application range of nano-size particles is constantly growing. Particles in the colloidal size range are used, e.g., as color pigments, to modify polymer properties, and in the cosmetic industry. This widespread application and thus increasing demand for these particle systems requires an efficient and economical production. For processes where the particles are obtained by precipitation, the solid-liquid separation is required for a change of solvent, for purification/washing of the particle system, obviously if the product is a powder or if a low solvent content is desired before thermal drying. So even if the product is a suspension, there still is the need for an effective solid-liquid separation during production.

The separation of colloidal particles is a challenging task. Due to the size of the particles, the mass forces can be neglected compared to the interparticulate forces. The prevailing particle interaction and large specific surfaces are accountable for many favorable effects and product properties of nano-size particles. The processing of these particle systems can at the

Correspondence: M. Hieke (mark.hieke@mvm.uni-karlsruhe.dc), Universität Karlsruhe, Institut für Mechanische Verfahrenstechnik und Mechanik, Straße am Forum, D-76131 Karlsruhe, Germany. same time become more laborious and time consuming. Separation by centrifugation requires very high $c$-values, which can only be achieved in fast rotating machines which also induce a considerable amount of shear force. This shear force can change the structure of aggregates and, along with that, the properties of the product. The special behavior of nano-scale particle systems leads to the question whether the well-known filter theory is applicable without alterations or whether it has to be adapted to the specific conditions.

Filtration of colloidal suspensions also faces several problems. Filter cakes of nano-size particles show very high cake resistances. This leads to very long filtration times and thus a small filter throughput, which has to be increased in order to keep up with the demand. In this study, we investigated the filtration behavior of comparatively thin filter cakes $(0.5-3 \mathrm{~mm})$ at different $\mathrm{pH}$ values and filtration pressures. By changing the $\mathrm{pH}$ value, the agglomeration tendency is changed as well. The filtration usually benefits from agglomeration by decreased filtration resistance and faster filtration. The agglomeration must be reversible so that the product properties can be restored. With, e.g., polymeric flocculants, the agglomeration would be irreversible and the properties would be changed permanently. The agglomeration by variation of the physico-chemistry, e.g. change of $\mathrm{pH}$ value or salt concentration, is for the most part reversible and the desired product properties are not altered by the separation process. 


\section{Materials and Methods}

\subsection{Materials}

In this study, we investigated the filtration behavior of Disperal 20 from Sasol. Disperal is a high-purity, highly dispersible, boehmite alumina powder. The dispersion of the Disperal powder was achieved by a combination of mechanical energy input by stirring and manipulation of interparticulate forces by variation of the $\mathrm{pH}$ value. The powder was dispersed in a $0.001-\mathrm{mol} / \mathrm{kg}$ potassium nitrate solution and nitric acid was added to set the $\mathrm{pH}$ value. All dispersions were prepared with a constant volume concentration of $5 \%$.

\subsection{Sample Preparation}

Colloidal particle systems are extremely sensitive to sample preparation. In order to yield reproducible results, a sample preparation method was developed and used for all experiments. A fixed sample volume was prepared for each experiment and the required amount for the specific experiment was taken from that batch. With the batch sample preparation, the amount of mechanical energy the agglomerates were exposed to is constant and reproducible results for different cake heights were obtained.

After weighing in the solids and liquids, the $\mathrm{pH}$ value was set by adding nitric acid. Then the sample was stirred using an Ultraturrax for $10 \mathrm{~min}$. Due to the heavy agitation, the sample was degassed at $10 \mathrm{kPa}$ for approximately $1 \mathrm{~min}$ to remove small air bubbles. As the suspensions had very low viscosities, the degassing time was set quite low. The sample needed to cool down to ambient temperature before the filtration started.

By this sample preparation method, we obtained suspensions with different agglomerate sizes. The use of an Ultraturrax with high shear forces results in comparatively rigid and dense agglomerates. In Fig. 1, the particle size distribution of the suspensions is displayed. The distributions were measured

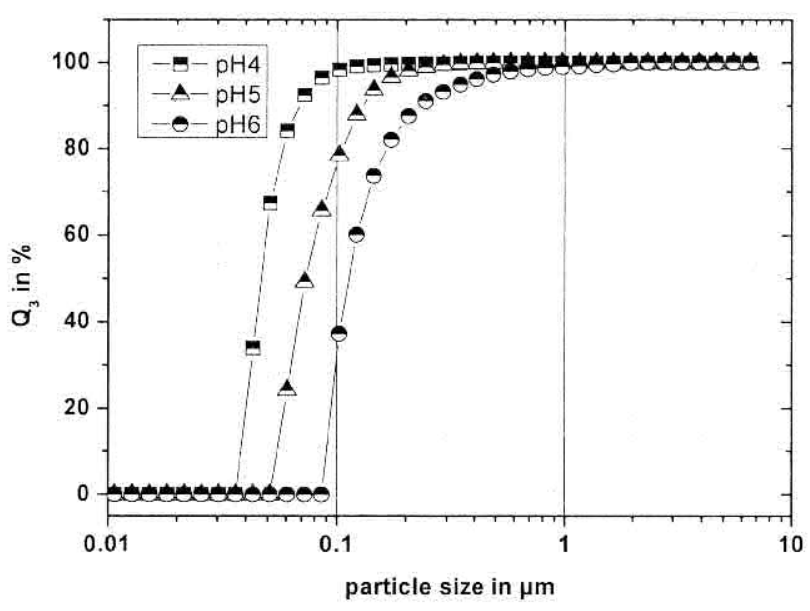

Figure 1. Cumulative particle size distribution. via dynamic light scattering with a Nanotrac from Microtrac, Inc. With increasing $\mathrm{pH}$ value, the repulsive particle-particle forces are reduced and larger agglomerates are formed.

Tab. 1 shows numerical values used to describe the particle size distributions. In this size range, the volume-specific value $M_{\mathrm{V}}$ is sensitive to very few larger particles. Few large particles do not influence the filtration behavior at all, but drastically skew the moments of a volume-specific size distribution. The $x_{3,50}$ and the number average $M_{\mathrm{A}}$ are much more significant for filtration behavior and show the expected tendency of increasing particle size with decreasing repulsive forces.

Table 1. Mean values of volume-specific particle size distribution.

\begin{tabular}{lclc}
\hline $\mathrm{pH}$ value & $x_{3,50}[\mathrm{~nm}]$ & $M_{\mathrm{V}}[\mathrm{nm}]$ & $M_{\mathrm{N}}[\mathrm{nm}]$ \\
\hline 4 & 46 & 225 & 51 \\
5 & 72 & 187 & 86 \\
6 & 113 & 1408 & 161 \\
\hline
\end{tabular}

\subsection{Nutsche Filtration}

The interpretation of filtration experiments is usually made according to the Darcy equation. The original equation is only valid for homogeneous packed beds that are formed by incompressible material.

Disperal 20 shows a certain degree of compressibility, in dependence on the $\mathrm{pH}$ value, but Darcy can be applied nevertheless. Due to the compressibility, the cake structure is no longer independent of the cake height. The porosity of the filter cake increases with the cake height. By using an average porosity for the calculation, one obtains a mean value of the cake resistance. This mean value can deviate significantly from the actual local porosity value within a certain layer.

$\frac{t}{V}=\frac{\eta \cdot r_{\mathrm{C}} \cdot \kappa}{2 A^{2} \cdot \Delta p} \cdot V+\frac{\eta \cdot R_{\mathrm{M}}}{A \cdot \Delta p}$

The concentration factor $\kappa$ is defined as:

$\kappa=\frac{c_{\text {sus }}}{1-\varepsilon-c_{\text {sus }}}$

Eq. (1) is a derivation of Darcy's filtration law, in which the overall resistance is split into the cake resistance $r_{\mathrm{C}}$ and the resistance of the filter media $R_{\mathrm{M}}$. This equation is very common in the interpretation of filtration data.

In order to obtain reproducible data, the device needed to be adapted to the special requirements. The filtration of thin filter cakes implies a very accurate height measurement. With cake heights of approximately $0.5 \mathrm{~mm}$, the determination with a dial indicator is not feasible. For this method, the filter cake has to be removed from the nutsche device, the filtration pressure has to be relieved, and the filter cake thus might increase in height. Another problem with this method is the mechanical stress implied by the measurement itself, which can result in a too low cake height. The piston of the dial can deform or even penetrate the filter cake surface, due to its elastic properties. 
For reliable and reproducible results, a non-contact, on-line optical method was used. A laser followed the sinking suspension level during filtration, providing the cake height at any time at filtration pressure. Thus, no correction or assumption for a change of height due to stress relief or cake handling is needed.

In Fig. 2, the effect of an error of $0.1 \mathrm{~mm}$ in the height measurement on the calculated resistance is displayed. For comparatively large filter cakes of $2 \mathrm{~mm}$ and more, the error due to the height measurement can be neglected. The smaller the sample, the more important is an accurate measurement; otherwise the resistance can deviate by a factor of 2 .

As mentioned before, the filtration experiments yield average values for the porosity and cake resistance as results. For a height-dependent analysis of the cake structure, the filter cake would have to be cut into layers. And these layers would have to be analyzed individually. Due to the small overall cake heights, this separation and further analysis is hardly feasible. For a height-dependent analysis, a non-invasive technique as described in Section 2.4 was used.

\subsection{Magnetic Resonance Imaging}

Magnetic resonance imaging (MRI) offers the possibility to non-invasively analyze the filter cake in situ in a sufficient resolution. This technique measures the proton density of a sample. In the aqueous suspensions investigated in this study, the main contribution to the signal came from water molecules; the signal of the solid content alone could be neglected. A more detailed description of MRI techniques used for engineering purposes can be found, e.g., in Hardy [1].

The used multi-spin multi-echo (MSME) measurements yielded a height resolution of $65 \mu \mathrm{m}$. With this resolution single agglomerates could not be resolved, but a change in subsequent layers could be monitored. $\Lambda$ single MSME experiment lasted approximately $40 \mathrm{~min}$; therefore, the filtration process had to be stopped. If the filtration continued, the structure and height of the filter cake would change during the measurement. To prevent a further filtration during the MRI experiment, the filtrate flux was interrupted by closing a valve.

In order to determine the porosity within a certain layer of the filter cake, the images obtained by the experiment had to be processed. The MSME experiment records the decay of the signal over time, so each time step yields an image with decreasing intensity. As mentioned before, the intensity of the signal is also dependent on the solid content $\phi$ of the sample. The signal decay or relaxation process follows an exponential function given in Eq. 2.

$I(\phi)=I_{0} \cdot(1-\phi) \cdot\left(1-e^{-\frac{v_{R}}{T_{1, \phi}}}\right) \cdot e^{-\frac{T_{E}}{T_{2, \phi}}}$

The echo time $T_{\mathrm{E}}$ and the repetition time $T_{\mathrm{R}}$ are parameters of the MSME sequence, and the relaxation times $T_{1, \phi}$ and $T_{2, \phi}$ are sample parameters. By a mono-exponential fit through the measured intensities $I(\phi)$, a pre-exponential factor (summarizing the effect of $\phi$ and $T_{1, \phi}$ ) and the decay rate $\frac{1}{T_{2, \phi}}$ can be determined. The decay rate proved to be very sensitive to changes

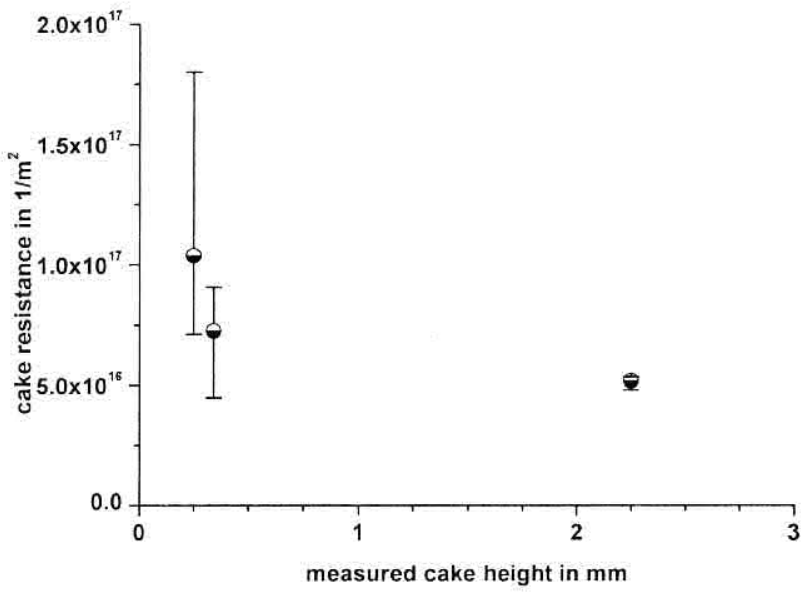

Figure 2. Influence of cake height measurement on cake resistances.

in the solid content of the sample, while the signal is almost noise free. The correlation of decay rate with solid content of the suspension or the filter cake, respectively, was obtained by calibration.

In the first step of data processing, the filter cake was cut from the image. The field of view of $20 \mathrm{~mm}$ in width and $16.6 \mathrm{~mm}$ in height included part of the filter cell as well as part of the drainage system, which would falsify the further processing. In the next step, the means of intensity for each line of voxels was calculated. This data was then fitted to Eq. 2 and the decay rate of each layer or voxel line was determined. The porosity of each layer was then calculated from the decay rate using the calibration function.

With the depicted filtration cell (see Fig. 3), suspensions can be filtrated within the tomograph with filtration pressures up to $300 \mathrm{kPa}$. By the in situ filtration, a change in structure due to handling and pressure relief is avoided.

For an analysis of the structure of a filter cake during the filtration process, the filtration was stopped with supernatant suspension. This was done to prevent a change of structure

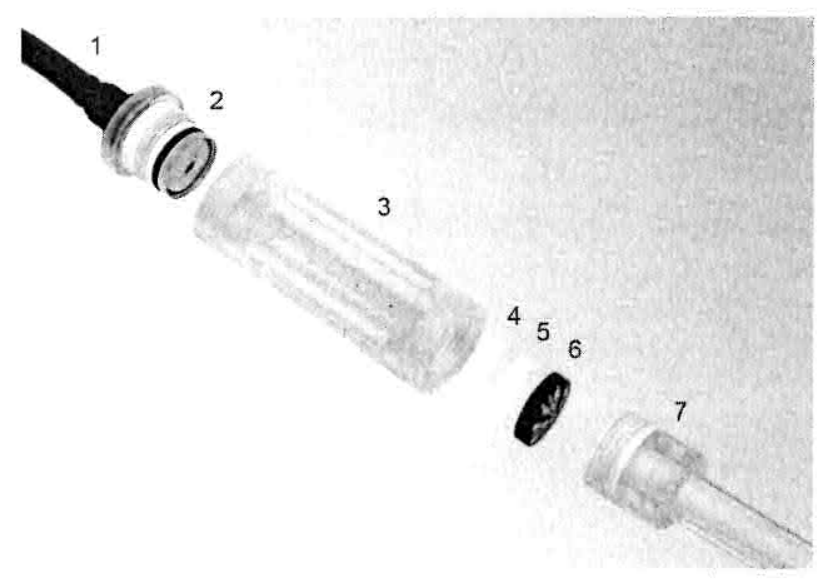

Figure 3. Filtration cell for use within NMR tomograph. (1) Hose, (2) lid, (3) cell, (4) filter membrane, (5) support media, $(6,7)$ drainage system including lower lid. 
due to partial consolidation of the filter cake. The consolidation process begins as soon as the top of the filter cake emerges from the suspension. During consolidation, the inhomogeneous cake structure of compressible filter cakes is restructured to a homogeneous pressure-dependent equilibrium state.

\section{Experimental Results}

\subsection{Data Obtained by Classical Nutsche Filtration}

Disperal formed compressible filter cakes during filtration. As mentioned before, one obtains only a mean overall value for porosity and overall filtration resistance. Neither the exact position where these values equal the actual ones nor the shape of the gradient within the structure can be resolved.

\subsubsection{Dependence of Porosity on $\mathrm{pH}$ Value}

In Fig. 4, the porosities in dependence on different overall cake heights are plotted for $\mathrm{pH}$ values of 4,5 and 6 at a constant filtration pressure. The porosities do not show a strong dependency on the $\mathrm{pH}$ value. The major part of the determined porosities is in the range of $70-75 \%$. By taking the scattering of the measured values into account, the porosities for a fixed $\mathrm{pH}$ value are independent of the cake height according to the theory. There seems to be only a weak dependence on the $\mathrm{pH}$ value. With increasing $\mathrm{pH}$ value, the porosities are also slightly increasing.

With the knowledge of filter cake porosity, a calculation of the filtration resistance is possible. The determined values of the height-specific mean filtration resistance $r_{\mathrm{C}}$ in dependence on the cake height are plotted in Fig. 5 for the different $\mathrm{pH}$ values. Compared to the small differences in porosity, the mean filtration resistances show a significantly stronger dependence on the $\mathrm{pH}$ value. The values for different cake heights are quite constant for each $\mathrm{pH}$ value. The smallest values were determined for filter cakes made from suspensions at $\mathrm{pH}$ 6. At

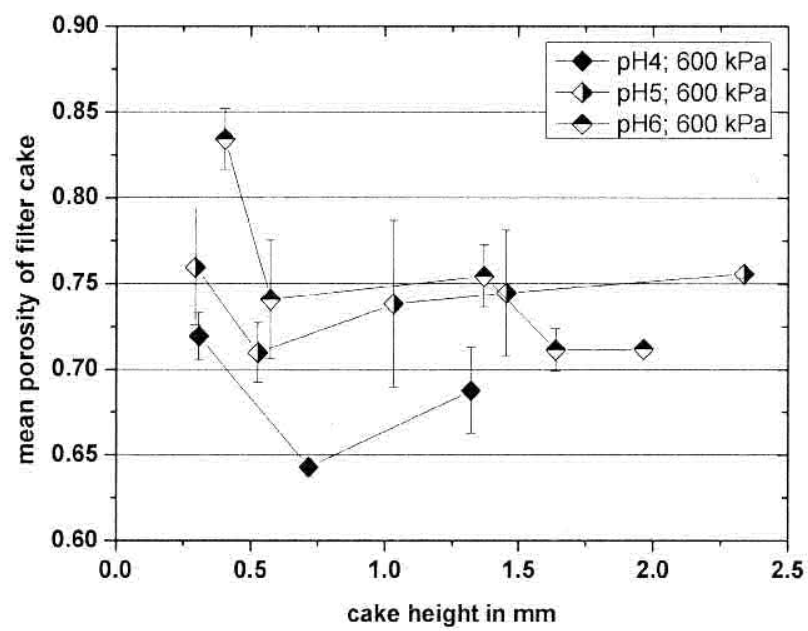

Figure 4. Porosities in dependence on the cake height plotted for different $\mathrm{pH}$ values. this $\mathrm{pH}$ value, the suspension had the largest mean particle size and the particles tended to form the largest agglomerates. With decreasing $\mathrm{pH}$ value, the agglomeration tendency decreased, also yielding more stabilized suspensions. In these suspensions, the repulsive forces were predominating, leading to smaller agglomerate sizes.

Small particles with repulsive interaction can be packed more densely than particles showing weak attractive forces. With attraction between particles, this attractive force has to be overpowered, once aggregates are in contact, in order to achieve a denser packing. The filtration resistance is determined by the filtrate which has to flow through the already built up filter cake. The filtrate flow through a filter cake made of agglomerates is less obstructed, because of larger pores between the agglomerates compared to the structure obtained by filtration of a stabilized suspension.

The small differences in porosity and considerable differences in filtration resistance can be explained with the suspension preparation. For homogenization, the suspension is stirred with a rotor/stator stirrer. This mechanism induces high shear forces. Agglomerates made with or exposed to high shear forces are very compact. Fragile agglomerates are frequently broken up in the high shear field and only densely packed agglomerates can withstand the shear force. So the agglomerates themselves show a low porosity comparable to a filter cake made from a stabilized suspension. With increasing agglomerate size, the pores between single agglomerates are larger, resulting in lower filtration resistances with comparable overall porosities.

\subsubsection{Dependence of Filtration Resistance on Filtration Pressure}

Fig. 6 shows the dependency of the height-specific mean filtration resistance on the filtration pressure. The mean resistance is again independent of the cake height. The dependency on the filtration pressure is weak compared to the influence of the

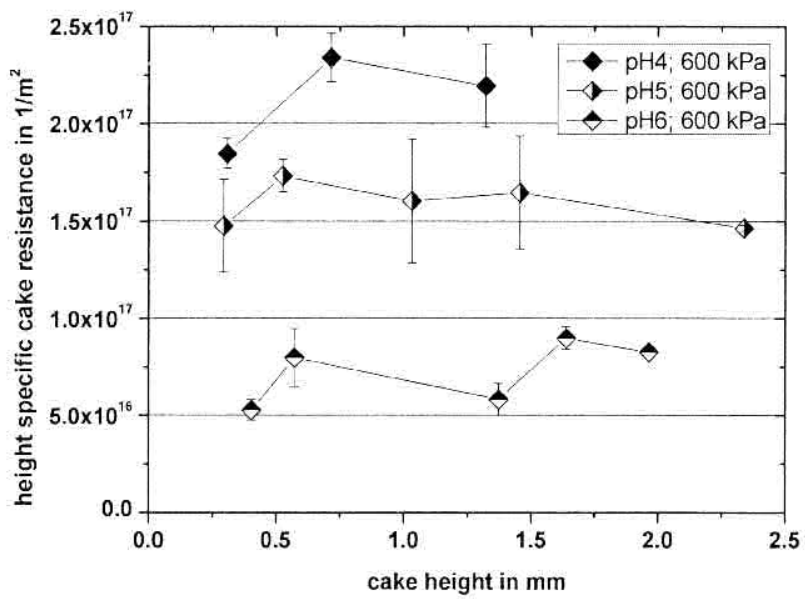

Figure 5. Mean filtration resistance in dependence on the cake height plotted for different $\mathrm{pH}$ values. 
$\mathrm{pH}$ value. The small pressure dependency can be explained by the small compressibility of the particle system. This can again be explained by the sample preparation. The compact agglomerates can withstand the mechanical load implied by the filtration pressure quite well. The weak links within the agglomerates were already broken in the high shear forces applied during preparation. So the compressibility is only due to the rearrangement of the agglomerates within the filter cake structure and the deformation of agglomerates can be neglected.

\subsubsection{Dependence of Filtration Time on $\mathrm{pH}$ Value}

The filtration times in dependence on the cake height are plotted in Fig. 7 for different pH values. For incompressible materials, the filtration time increases with the square of the cake height. For compressible materials, the exponent will increase. The conducted experiments yielded exponents between 2.1 and 2.5 .

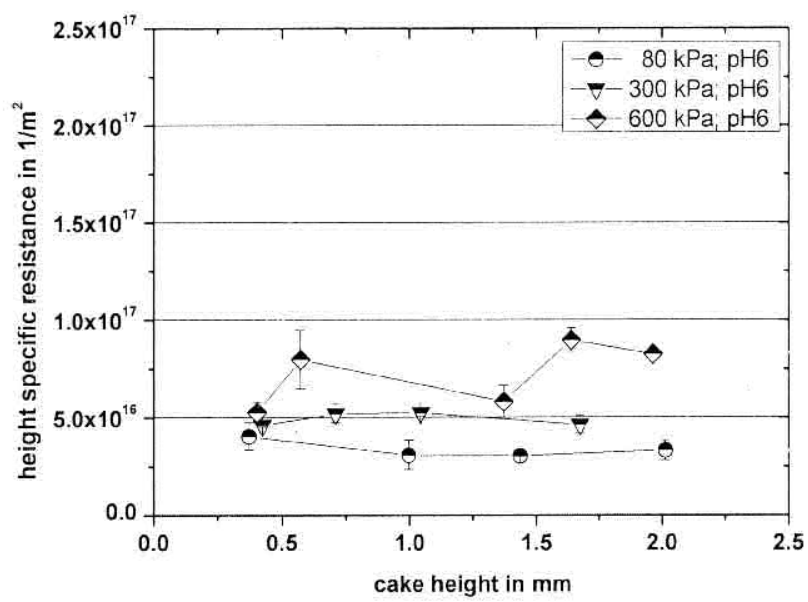

Figure 6. Mean filtration resistances in dependence on the cake height plotted for different filtration pressures.

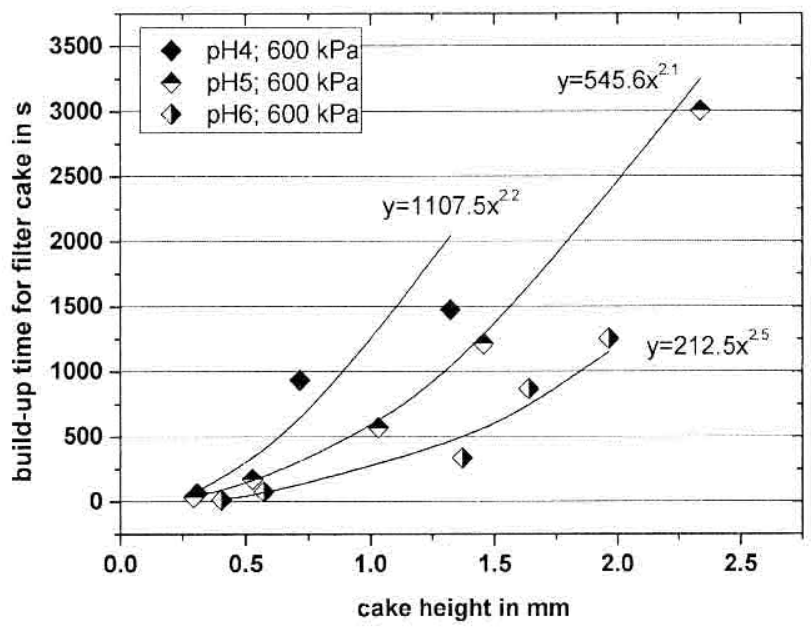

Figure 7. Filtration time in dependence on the $\mathrm{pH}$ value plotted for different cake heights.
The filtration time shows the expected tendency. For a filtration with the largest agglomerates and thus highest compressibility, we observed the fastest filtration and highest exponent. For the suspensions of smaller agglomerates, the filtration time was significantly longer. The longest filtration times were observed with stabilized suspensions.

\subsection{Determination of Local Porosity by MRI}

MRI techniques enable us to measure the actual porosity in different cake layers. With this non-invasive technique, a height resolution of $65 \mu \mathrm{m}$ is achieved.

Fig. 8 shows the porosity gradient within a filter cake made by a suspension at $\mathrm{pH} 4$ and a filtration pressure of $300 \mathrm{kPa}$. The porosities for two different filter cakes are plotted in this figure. The circles represent the porosity obtained during filtration; the diamonds represent a filter cake that was consolidated after filtration. Filtration of compressible material results in an inhomogeneous structure. If we look at the circles in Fig. 8, we can clearly see an unambiguous dependency of porosity on cake height. The overall cake height was $1.4 \mathrm{~mm}$; the constant values of 0.95 for higher cake heights are measured in the supernatant suspension. The shape of the porosity gradient can be divided into two regimes. Right at the filter media, the porosity has the lowest measured value of 0.59 and is then rising slowly to a value of 0.71 at a cake height of $0.9 \mathrm{~mm}$. At this point, the gradient becomes much steeper and rises to the suspension concentration at the very top of the filter cake. Tiller and Shirato [2] predicted similar shapes for the porosity over height for compressible materials.

There is a significant gradient observable within the filter cake. Disperal 20 thus is a compressible material, but it does not form a super-compressible skin layer reported for certain materials. This skin layer forms right next to the filter media and, due to the extreme compressibility, has a very high filtration resistance. Almost all of the filtration pressure is dissipated in this layer, and for subsequent layers, only a small frac-

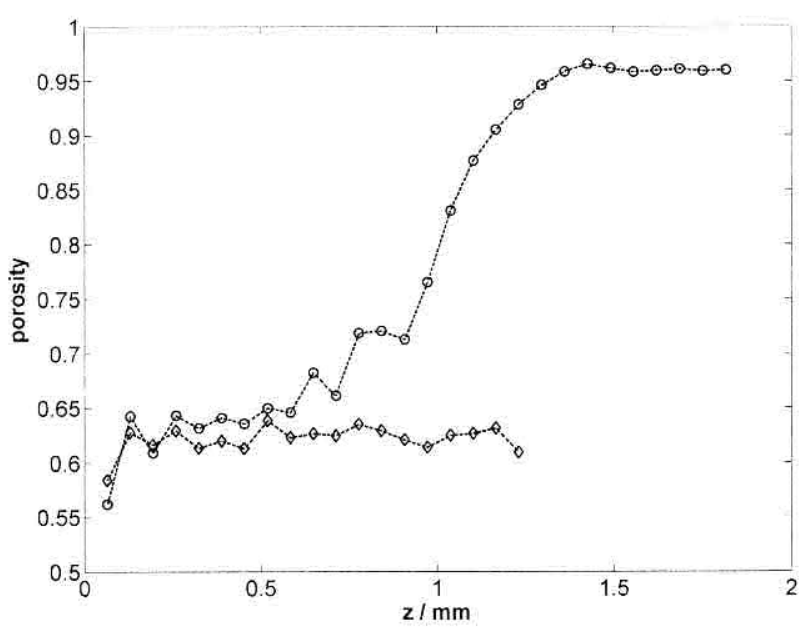

Figure 8. Porosity of a non-consolidated (supernatant suspension) and a consolidated filter cake at $300 \mathrm{kPa}$ and $\mathrm{pH} 4$. 
tion of the applied pressure difference is left. This leads to almost constant high porosities for the rest of the cake as soon as the skin layer has formed. The porosity gradient for a skin layer model would show a small porosity for the skin layer near to the filter media, followed by a sudden increase to a significantly higher porosity, with a further moderate increase from this point to suspension concentration within the filter cake. This behavior was reported, e.g., by Tiller and Green [3], Alles [4] and Anlauf and Erk [5].

The diamonds in Fig. 8 represent the porosity gradient for filter cakes obtained by another experiment. In this experiment, an identical suspension volume was filtrated, but the filtration was not stopped during cake filtration. The filtration was continued until the filter cake was consolidated. During consolidation, the filter cake is compressed by the applied filtration pressure. The gas is acting like a piston since the capillary entry pressure of the filter cake has not been overcome. This compression reaches a pressure-dependent equilibrium, where the porosity of the filter cake is homogeneous over the height.

For these filtration parameters, the consolidation porosity is slightly above 0.60 . This is the same value we saw during filtration in the very first layers of the filter cake. If we had a skin layer and it could not be resolved with our resolution during filtration, we would have observed a discrepancy between filtration and consolidation values. As mentioned before, the porosities of the filter cake are reduced to the pressure-dependent consolidation porosity, which would be equal to the porosity of the skin layer. If the resolution was too coarse, the lowest measured porosities in the filtration experiment would have shown higher values compared to the consolidation porosity. The resolution of the MRI measurement thus is sufficiently high to resolve all relevant effects in this particle system.

Fig. 9 shows the porosity gradient for different cake heights with otherwise constant filtration parameters. $\Lambda$ t all heights, the shape of the gradient is similar. In the bottom part, we see a nearly consolidated filter cake with a slowly rising porosity which is followed by a steep gradient in the upper layers. This slope seems to decrease with increasing overall cake height, so the compaction process of the lower part of the filter cake takes longer than the filtration of new particles from the suspension.

A further validation of the new technique can be achieved by a comparison of classical filtration data with the results from the MRI experiments. The classical filtration experiments yielded a mean porosity of $74 \%$ for filtration at $300 \mathrm{kPa}$ and $\mathrm{pH}$ 4. The means of porosity calculated from the MRI experiments depicted in Fig. 9 show values between 71 and $73 \%$. The two different measuring techniques are in good agreement; the new MRI analysis proved to be a reliable and reproducible way to determine the porosity gradient within a filter cake.

\section{Conclusions}

The filtration behavior of colloidal suspensions is affected by many variables. In this paper, the influence of interparticulate interaction and filtration pressure on the filtration process was

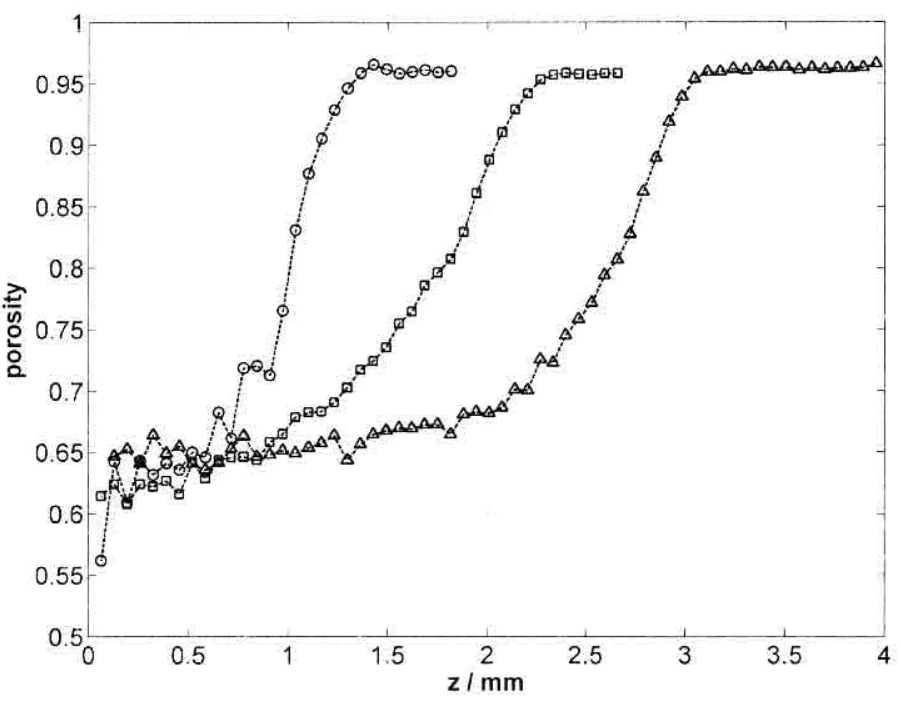

Figure 9. Porosity for filter cakes with different overall cake heights at $300 \mathrm{kPa}$ and $\mathrm{pH} 4$

studied. The determination of the local porosity gradient in thin cake layers was accomplished by MRI techniques.

This study found that:

- The global mean porosity and the mean porosity from the local porosity gradient are in good agreement.

- The resolution of the MRI measurement is sufficiently high to resolve all relevant effects. The virtual layers are adequately homogeneous for an interpretation of the filtration.

- The mean height-specific filtration resistances proved to be independent of the cake height.

- The filtration times showed a strong dependency on the interparticulate interaction. The filtration time increases with the cake height up to the power of 2.5 for the regarded suspensions.

- The local porosities show a similar shape for different cake heights, but with increasing cake height the slope decreases. This larger area with high porosities requires a longer consolidation time compared to smaller cake heights.

For a filtration of colloidal suspensions, the approach of a filtration in comparatively thin layers shows promise. Larger filter cakes show the same mean values of filtration resistance and mean porosity, but require a disproportionately longer filtration time.

\section{Acknowledgements}

Financial support from the Deutsche Forschungsgemeinschaft (DFG) within the project SPP-1273 is gratefully acknowledged.

\section{Symbols used}

$\begin{array}{lll}A & {\left[\mathrm{~m}^{2}\right]} & \begin{array}{l}\text { filtration area } \\ \text { volume concentration of } \\ \text { suspension }\end{array}\end{array}$




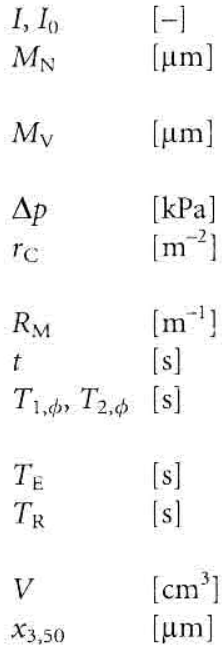

NMR signal intensity number-specific moment of particle size distribution volume-specific moment of particle size distribution

filtration pressure

height-specific filtration resistance

resistance of the filter media time

relaxation times of sample in NMR

echo time in NMR experiment repetition time in NMR experiment volume characteristic value of particle size distribution
Greek symbols

$\begin{array}{lll}\varepsilon & {[-]} & \text { porosity } \\ \eta & {[\mathrm{Pa} \mathrm{s}]} & \text { dynamic viscosity } \\ \kappa & {[-]} & \text { concentration factor } \\ \phi & {[-]} & \text { solid content }\end{array}$

\section{References}

[1] E. H. Hardy, Chem. Eng. Technol. 2006, 29 (7), 785.

[2] F. M. Tiller, M. Shirato, AIChE J. 1964, 10, 61.

[3] F. M. Tiller, T. C. Green, AIChE J. 1973, 19, 1266.

[4] C. Alles, Ph.D. Thesis, Universität Karlsruhe (TH) 2000.

[5] H. Anlauf, A. Erk, AufbereitungsTechnik 2006, 47 (3), 22. 